\title{
Factors Affecting the Induction of Lignin Peroxidase in Manganese-Deficient Cultures of the White Rot Fungus Phanerochaete chrysosporium
}

\author{
Avi Matityahu1,2, Aya Sitruk1, Yitzhak Hadar³, Paula A. Belinky ${ }^{\text {* }}$ \\ ${ }^{1}$ Tel Hai Academic College, Kiryat Shmona, Israel \\ ${ }^{2}$ Faculty of Medicine in the Galilee, Bar-Ilan University, Safed, Israel \\ ${ }^{3}$ Department of Plant Pathology and Microbiology, The Robert H. Smith Faculty of Agriculture, Food and \\ Environment, The Hebrew University of Jerusalem, Rehovot, Israel \\ Email: ${ }^{*}$ paudabe@gmail.com
}

Received 19 January 2015; accepted 6 February 2015; published 10 February 2015

Copyright (C) 2015 by authors and Scientific Research Publishing Inc.

This work is licensed under the Creative Commons Attribution International License (CC BY). http://creativecommons.org/licenses/by/4.0/

(c) (7) Open Access

\begin{abstract}
The lignin peroxidase (LIP) production and regulation, in manganese ions $\left(\mathrm{Mn}^{2+}\right)$ deficient cultures of the white rot fungus Phanerochaete chrysosporium, is still not clearly understood. Mn $^{2+}$ deficiency is correlated to low levels of manganese containing superoxide dismutase (MnSOD). In this work, we show that despite the low activity level of MnSOD in $\mathrm{Mn}^{2+}$-deficient cultures, the presence of $\mathrm{H}_{2} \mathrm{O}_{2}$ is essential for the expression of the lip- $\mathrm{H} 2$ gene, which encodes for the major LIP isoenzyme produced (LIP-H2). Thus, the $\mathrm{H}_{2} \mathrm{O}_{2}$ present in $\mathrm{Mn}^{2+}$-deficient cultures is probably produced by other mechanisms rather than dismutation of superoxide ions by MnSOD. Glyoxal oxidase gene (glox) expression was significantly higher than MnSOD (MnSOD1) and cellobiose dehydrogenase (cdh1) expression in $\mathrm{Mn}^{2+}$-deficient cultures, indicating its clear involvement in $\mathrm{H}_{2} \mathrm{O}_{2}$ production in those cultures. Glyoxal oxidase may compensate the absence of MnSOD activity in $\mathrm{Mn}^{2+}$-deficient cultures. The high levels of reactive oxygen species (ROS) needed for the enhancement of LIP expression in $\mathrm{Mn}^{2+}$-deficient cultures were not directly correlated to the protein kinase C (PKC) activity involved in signal transduction pathway. High level of oxidative stress was observed in MnSOD silenced mutants, grown in the presence of $\mathrm{Mn}^{2+}$, indicating that oxidative stress in $\mathrm{Mn}^{2+}$-deficient cultures was caused by low levels of MnSOD rather than the deficiency in $\mathrm{Mn}^{2+}$. The results of this work can further contribute to the understanding of LIP regulation in $\mathrm{Mn}^{2+}$-deficient cultures.
\end{abstract}

\footnotetext{
${ }^{*}$ Corresponding author.
}

How to cite this paper: Matityahu, A., Sitruk, A., Hadar, Y. and Belinky, P.A. (2015) Factors Affecting the Induction of Lignin Peroxidase in Manganese-Deficient Cultures of the White Rot Fungus Phanerochaete chrysosporium. Advances in Microbiology, 5, 83-92. http://dx.doi.org/10.4236/aim.2015.52009 


\section{Keywords}

\section{ROS, GLOX, MnSOD, PKC, lip-H2 Induction}

\section{Introduction}

Lignin is an amorphous and insoluble polymer lacking stereoregularity, which plays a key role in the carbon cycle as the most abundant aromatic compound and as a protective matrix surrounding the cellulose microfibrils of plant cell walls [1] [2]. Fungi collectively referred to as white rot basidiomycetes are the only microbes capable of efficiently depolymerizing and mineralizing this recalcitrant polymer [3] [4]. The most intensively studied white rot fungus, Phanerochaete chrysosporium, secretes an array of peroxidases that act via the generation of aromatic free radicals, which undergo spontaneous cleavage reactions. Two major families of hydrogen peroxide $\left(\mathrm{H}_{2} \mathrm{O}_{2}\right)$-requiring extracellular heme-peroxidases designated lignin peroxidase (LIP) and manganese-dependent peroxidase (MNP) were identified [5]. The non-specific nature and exceptional oxidation potential of the LIP have attracted considerable interest in organopollutants degradation and fiber bleaching. The regulation of the gene families encoding extracellular peroxidases is poorly understood, but it is clear that oxidative stress is a key factor [6]-[8].

LIP expression requires nutrient or carbon starvation, signaled by a rise in intracellular cAMP concentrations [9] and concomitant pulses of pure oxygen gas in the headspace. Starving cultures without excessive oxygen are not enough for triggering LIP expression, indicating that both effects need to be simultaneous [10]-[15]. The effect of such elevated oxygen concentrations on LIP expression can be reproduced by depleting manganese ions $\left(\mathrm{Mn}^{2+}\right)$ from cultures, in the presence of atmospheric air [7] [8]. Development of a putative oxidative stress is detected in LIP-producing cultures of $P$. chrysosporium, either oxygenated or $\mathrm{Mn}^{2+}$-deficient. This was evidenced by measuring high levels of reactive oxygen species (ROS) with specific molecular probes, and was further confirmed by the enhancement of the antioxidant defense system and by the high degree of oxidative damage of major macromolecules, lipids, protein and DNA. In oxygenated cultures, increased expression of manganese superoxide dismutase (MnSOD) was the major response of the antioxidant system. In contrast, in $\mathrm{Mn}^{2+}-$ deficient cultures, negligible activity of MnSOD was detected [6] [7].

The existence of oxidative stress in LIP-producing cultures led to the hypothesis that ROS, and hydroxyl radical $\left(\mathrm{OH}^{\circ}\right)$, in particular, may act as intracellular messengers, triggering lip- $H 2$ expression (the major LIP enzyme expressed) in liquid cultures of $P$. chrysosporium, through signal transduction [6] [7]. In a previous work, we found that addition of a $\mathrm{OH}^{*}$ scavenger, dimethylsulfoxide (DMSO), to the cultures, completely abolished LIP transcription (both mRNA and heme-protein were undetectable), indicating that these ROS, coupled to high levels of cAMP, were indeed involved in the induction of lip- $\mathrm{H} 2$ expression. This effect was confirmed by in-situ generation of $\mathrm{OH}^{*}$ via the addition of Fenton reagents ( $\mathrm{OH}^{*}$ producers), which significantly enhanced lip-H2 expression [6] [7] [16].

Significantly lower protein kinase C (PKC) activity in oxygenated vs. aerated (grown with free exchange of atmospheric air) low-LIP producing cultures was measured [16]. In oxygenated cultures, inactivation of PKC activity by calphostin C, staurosporin A or H7 (PKC activity inhibitors) caused significant elevation in lip-H2 and MnSOD1 expression. Stimulation of PKC by phorbol 12-myristate 13-acetate (PMA) caused the reverse effect [16].

Significantly low levels of lip- $\mathrm{H} 2$ expression were detected when superoxide anion $\left(\mathrm{O}_{2}^{--}\right), \mathrm{H}_{2} \mathrm{O}_{2}$ or $\mathrm{OH}^{*}$ was scavenged by 4,5-dihydroxy-1,3-benzene disulfonic acid (tiron), pyruvate or DMSO, respectively, whether the level of PKC was normal, stimulated or inactivated [16]. In addition, in-situ generation of $\mathrm{OH}^{*}$, via addition of Fenton reagents to aerated cultures, reduced $p k c$ expression. In contrast, $\mathrm{OH}^{*}$ scavenging stimulated $p k c$ expression [16]. It was suggested that due to high $\mathrm{OH}^{*}$ levels, fungal cells activated a complex defensive system which regulated the levels of Fenton components by repressing PKC and stimulating LIP, MnSOD1 and catalase [16].

Since the difference between both types of LIP-producing cultures seems to partially relay on inactivation or lack of MnSOD activity, it is possible that ROS production occurs by different mechanisms. MnSOD product, $\mathrm{H}_{2} \mathrm{O}_{2}$, might be produced by glyoxal oxidase (GLOX) or cellobiose dehydrogenase (CDH) present in the fungus [17] in $\mathrm{Mn}^{2+}$-deficient cultures. To clarify if deficiency of $\mathrm{Mn}^{2+}$ provokes the generation of high ROS concentra- 
tions only via the lack in MnSOD activity, MnSOD silenced mutants (MSC) of P. chrysosporium were previously prepared by using siRNA technique [18]. Significantly lower MnSOD expression, at both the mRNA and protein activity levels, was detected in MSC mutants [18].

In this work, we try to explain how $\mathrm{Mn}^{2+}$ deficiency in cultures of $P$. chrysosporium causes enhancement of lip- $\mathrm{H} 2$ expression in relation to oxidative stress. The results of this work can contribute to the overall understanding of LIP regulation in white rot fungi.

\section{Materials and Methods}

\subsection{Reagents}

Veratryl alcohol, phenylmethanesulfonyl fluoride (PMSF), pyruvate, staurosporin A, calphostin C, H7, leupeptin, aprotinin, ethylenediaminetetraacetic acid (EDTA), N,N,N',N'-tetramethylethylenediamine (TEMED), 4,5-dihydroxy-1,3-benzene disulfonic acid (Tiron), phosphinothricin (PPT), riboflavin 5,5'-dithiobis-2-nitrobenzoic acid (DTNB), 2',7'-dichlorodihydrofluorescein (DCF) and Tri Reagent were purchased from Sigma (St. Louis, MO). PepTag non-radioactive kit was purchased from Promega (Madison, WI). Reverse-IT ${ }^{\text {TM }} 1^{\text {st }}$ Strand Synthesis kit and Syber Green were purchased from Abgene (Epsom, UK).

\subsection{Fungal Strain and Culture Conditions}

The filamentous fungus $P$. chrysosporium Burds BKM-F-1767 (ATCC 24725) was used for this study. The fungus was maintained at $4{ }^{\circ} \mathrm{C}$ on $2 \%(\mathrm{wt} / \mathrm{vol})$ malt extract agar slants and inoculated by the method of Tien and Kirk [19]. The growth medium was prepared as previously described [8] [20] with initial concentrations of glucose, diammonium tartrate and $\mathrm{MnSO}_{4} \cdot \mathrm{H}_{2} \mathrm{O}$ of 56, 2.4 and $0.225 \mathrm{mM}$, respectively. Two different cultures of the fungus were studied: aerated cultures: The fungus was grown in submerged liquid cultures $(90 \mathrm{ml})$ at 175 $\mathrm{rpm}$ at $37^{\circ} \mathrm{C}$ in $250-\mathrm{ml}$ flasks with free exchange of atmospheric air (flasks sealed with dense-paper plugs). $\mathrm{Mn}^{2+}$-deficient cultures: The fungus was grown at the same culture conditions as above, but with depletion of $\mathrm{Mn}^{2+}$. Unless otherwise specified, the cultures were incubated for 5 days.

MSC mutants preparation was described in a previous work using siRNA silencing technique [18]. The MSC mutants were grown in oxygenated cultures, containing $0.225 \mathrm{mM} \mathrm{MnSO}_{4} \cdot \mathrm{H}_{2} \mathrm{O}$. The MSC mutants were grown in submerged liquid cultures $(90 \mathrm{ml})$ at $175 \mathrm{rpm}$ at $37^{\circ} \mathrm{C}$ in 250 -ml flasks sealed with rubber stoppers, and the headspace was flushed twice a day with $\mathrm{O}_{2}$ for $2 \mathrm{~min}$ at a flow rate of $1 \mathrm{l} / \mathrm{min}$. Oxygen gas was of medical-grade purity.

\subsection{Measurement of Enzyme Activities}

LIP activity was measured in the extracellular medium of the fungus culture. PKC and catalase activities were measured in protein extracts. For preparation of protein extracts, fungal pellets, harvested after $120 \mathrm{~h}$, were separated from extracellular fluid by filtration through cheesecloth, and the biomass was frozen at $-80^{\circ} \mathrm{C}$. Biomass samples were ground with a mortar and pestle in the presence of liquid nitrogen. The powder was then suspended in $0.5 \mathrm{ml}$ of cold extraction buffer, $25 \mathrm{mM}$ Tris- $\mathrm{HCl}$ (pH 7.4), $0.5 \mathrm{mM}$ EDTA, $0.5 \mathrm{mM}$ EGTA, 0.05\% Triton X-100, $10 \mathrm{mM} \beta$-mercaptoethanol, $1 \mu \mathrm{g} / \mathrm{ml}$ leupeptin, $1 \mu \mathrm{g} / \mathrm{ml}$ aprotinin, $0.5 \mathrm{mM}$ PMSF and $200 \mathrm{mM}$ $\mathrm{NaCl}$. The lysates were centrifuged at $14,000 \times g$ for $5 \mathrm{~min}$ at $4^{\circ} \mathrm{C}$.

\subsection{LIP Activity}

LIP activity was measured according to the method of Tien and Kirk [19]. The oxidation of veratryl alcohol to veratryl aldehyde was recorded at $310 \mathrm{~nm}$ for $40 \mathrm{~s}$, with a unit being defined as $1 \mu \mathrm{mol}$ of veratryl alcohol oxidized to veratryl aldehyde per minute.

\subsection{PKC Activity}

Samples of protein extracts ( $5 \mu \mathrm{g}$ ) from the different experimental conditions tested were assayed for PKC activity using the PepTag non-radioactive kit (Promega) according to the manufacturer's directions. This kit uses the brightly colored fluorescent peptide substrate PLSRTLSVAAK. The hot pink color is imparted by a dye molecule conjugated to the substrate. The reaction was performed for $30 \mathrm{~min}$ at $30^{\circ} \mathrm{C}$. The non-phosphorylated 
peptide substrate was then separated from the phosphorylated substrate by electrophoresis on a $0.8 \%$ agarose gel, according to their migration to the anode and cathode, respectively. The gels were photographed on a transilluminator. PKC activity was estimated by the fluorescence intensity of the band corresponding to the non-phosphorylated substrate. More intensive band was corresponding to low PKC activity. The densities of the areas of activity were measured and compared by using TINA program software (Raytest Isotopenmessgeräte GmbH).

\subsection{Catalase Activity}

Catalase activity was assayed by measuring the degradation of $\mathrm{H}_{2} \mathrm{O}_{2}$. The rate of disappearance of $\mathrm{H}_{2} \mathrm{O}_{2}$ was monitored at an absorbance of $240 \mathrm{~nm}$ [21]. One unit of catalase decomposed 1 mol of $\mathrm{H}_{2} \mathrm{O}_{2}$ in 1 min $\left(\varepsilon_{\mathrm{H}_{2} \mathrm{O}_{2}}=\right.$ 39.4 $\left.\mathrm{M}^{-1} \mathrm{~cm}^{-1}\right)$.

\subsection{Influence of $\mathrm{H}_{2} \mathrm{O}_{2}$ on the Induction of lip- $\mathrm{H} 2$ Expression}

To determine the effect of $\mathrm{H}_{2} \mathrm{O}_{2}$, on lip- $\mathrm{H} 2$ expression, the fungus was grown under the conditions described above, with or without the addition of a $\mathrm{H}_{2} \mathrm{O}_{2}$ scavenger, pyruvate, at different concentrations (0.1 - $\left.5 \mathrm{mM}\right)$, at $72 \mathrm{~h}$, and at $96 \mathrm{~h}$. lip- $\mathrm{H} 2$ expression from cultures incubated with or without pyruvate was measured as described in Section 2.7. $\mathrm{H}_{2} \mathrm{O}_{2}$ levels in $120 \mathrm{~h}$ liquid cultures of $P$. chrysosporium were detected by adding 2',7'-dichlorodihydrofluorescein (a fluorescent indicator of peroxide). This compound was added to the cultures at a final concentration of $30 \mu \mathrm{M}$ and incubated for $30 \mathrm{~min}$ at $37^{\circ} \mathrm{C}$ and $250 \mathrm{rpm}$. The mycelia were then harvested, washed with double-distilled water, and treated with $2 \mathrm{ml}$ of 5\% 5-sulfosalicylic acid (vol/vol) for 20 $\min$ at $4^{\circ} \mathrm{C}$ and a cellular extract was obtained. The treated mycelia were centrifuged at $20,000 \times g$ for $15 \mathrm{~min}$ at $4^{\circ} \mathrm{C}$. The production of 2',7'-dichlorofluorescein was measured in the extracellular medium and in the cellular extract with a spectrofluorimeter (Victor ${ }^{3}$, PerkingElmer, USA). To detect 2',7'-dichlorofluorescein, the extinction and emission wavelengths were 501 and $521 \mathrm{~nm}$.

\subsection{Influence of PKC on the Induction of lip-H2 Expression}

To determine the effect of PKC on lip-H2 expression, the fungus was grown for $120 \mathrm{~h}$ under the conditions described above, then PKC inhibitors were added, or not, for 2 h. The PKC inhibitors staurosporin A, calphostin C or $\mathrm{H} 7$ were added at concentrations of $40 \mathrm{ng} / \mathrm{ml}, 122 \mathrm{ng} / \mathrm{ml}$ or $5 \mu \mathrm{g} / \mathrm{ml}$, respectively. lip- $H 2$ expression from cultures incubated with or without PKC inhibitors were measured as described below.

\subsection{Measurement of glox, MnSOD1, cdh1 and lip-H2 Expression by Real-Time PCR}

Frozen biomass was ground with a mortar and pestle in the presence of liquid nitrogen. Total RNA was extracted from each sample using Tri Reagent (Sigma). Total cDNA was generated by reverse transcription using the Reverse-IT ${ }^{\mathrm{Tm}} 1^{\text {st }}$ Strand Synthesis kit (ABgene, Epsom, UK). The amount of each gene in relation to $18 S$ rRNA transcript was determined by real-time PCR, based on the high-affinity double-stranded DNA-binding dye Syber Green (Absolute ${ }^{\mathrm{TM}}$ QPCR SYBER ${ }^{\circledR}$ Green ROX mix, ABgene, Epsom, UK). Each reaction was performed in triplicate in a spectrofluorometric thermal cycler (Rotor-Gene ${ }^{\mathrm{TM}}$ 3000, Corbett Research, Sydney, Australia), using the primers AM42, AM43 for 18S rRNA, AM63, AM64 for MnSOD1, AM44, AM45 for lip-H2, AM83, AM84 for glox and AS114, AS115 for cdh1 (Table 1). The real-time PCR program included a 15 -min polymerase activation step at $95^{\circ} \mathrm{C}$ followed by up to 45 cycles of $15 \mathrm{~s}$ at $95^{\circ} \mathrm{C}, 20 \mathrm{~s}$ at the optimal annealing temperature $\left(60^{\circ} \mathrm{C}\right)$ and $25 \mathrm{~s}$ at $72^{\circ} \mathrm{C}$. Assay specificity was confirmed by subjecting the PCR products to SYBER Green I melting curves. The efficiency of real-time amplification was determined by running a standard curve with serial dilution of cDNA and defined as $\mathrm{E}=[10(-1 / \mathrm{m})]-1(\mathrm{~m}=$ slope of reaction). Optimal melting points for $18 \mathrm{~S} r R N A, M n S O D 1$, lip- $H 2$, glox and $c d h 1$ were $84^{\circ} \mathrm{C}, 93^{\circ} \mathrm{C}, 91^{\circ} \mathrm{C}, 89^{\circ} \mathrm{C}, 90.5^{\circ} \mathrm{C}$ and $84^{\circ} \mathrm{C}$, respectively. The reaction efficiencies for $18 S$ rRNA, MnSOD1, lip-H2, glox and $c d h 1$ were 93\% $\left(\mathrm{R}^{2}=0.999\right), 80 \%$ $\left(\mathrm{R}^{2}=0.994\right)$, 85\% $\left(\mathrm{R}^{2}=0.997\right), 97 \%\left(\mathrm{R}^{2}=0.998\right), 99 \%\left(\mathrm{R}^{2}=0.999\right)$ and $84 \%\left(\mathrm{R}^{2}=0.991\right)$ respectively.

\subsection{Determination of Oxidative Damage to Macromolecules}

2.10.1. Measurements of Oxidative Damage

Oxidative damage was determined by detecting the levels of oxidized lipids with LPO-586 kit (Oxis Research) 
Table 1. Primers used in this study.

\begin{tabular}{lcc}
\hline & Description & Sequence (5'-3') \\
\hline AM42 & $18 S r R N A$ reverse & CAACTACGAGCTTTTTAACTGC \\
AM43 & $18 S r R N A$ forward & CAAATTACCCAATCCCGACAC \\
AM44 & $l i p-H 2$ forward & GGCAGTCCTTCGTCAACAAC \\
AM45 & $l i p-H 2$ reverse & ATGTCGGCGTGCGTCTTAC \\
AM63 & MnSOD 1 reverse & GCTCTAGAGAGCCAGCCCCAGCC \\
AM64 & MSOD 1 forward & CGGGATCCATGTCCGGCCAGCACAC \\
AM83 & glox forward & GACCCTGCGACTGTTCAT \\
AM84 & glox reverse & AGCGACGATAAAGACGG \\
AS114 & $c d h 1$ forward & CGTTTTCCCCCCTCT \\
AS115 & $c d h 1$ reverse & TCCGCCGCCATTG \\
\hline
\end{tabular}

following the manufacturer's directions. This kit detects a chromogen that absorbs at 586 nm and is formed by the reaction between malondialdehyde (MDA), an end product of the peroxidation of polyunsaturated fatty acids, and N-methyl-2-phenylindole.

\subsubsection{Reduced Glutathione:Oxidized Glutathione (GSH:GSSG) Ratio}

The GSH/GSSG molar ratio was determined with a Glutathione assay kit (Sigma) according to the manufacturer's directions, with slight modifications. This method employs 5,5'-dithiobis-2-nitrobenzoic acid (DTNB), which reacts with GSH to form 2-nitro-5-thiobenzoate anion ( $\mathrm{TNB}^{2-}$ ), a product which can be detected spectrophotometrically at $412 \mathrm{~nm}$ with an extinction coefficient of $14,150 \mathrm{M}^{-1} \mathrm{~cm}^{-1}$. The concentration of GSH was calculated from a calibration curve (0 - $3 \mathrm{M} \mathrm{GSH).} \mathrm{The} \mathrm{assay} \mathrm{was} \mathrm{conducted} \mathrm{on} \mathrm{two} \mathrm{parallel} \mathrm{sets} \mathrm{of} \mathrm{samples.}$ One set, preincubated with glutathione reductase, gave total glutathione (reduced and oxidized) as GSH. The second set, used for GSSG determination, was first treated with the thiol-scavenging reagent 1-methyl-2-vinylpyridinium trifluoromethanesufonate to scavenge GSH. The remaining GSSG was then reduced to GSH with NADPH catalyzed by glutathione reductase and the formed GSH was measured. GSSG at concentrations from 0 to $1.5 \mathrm{M}$ was used as the standard to calibrate the curves. The GSH/GSSG ratio was calculated as follows: $\mathrm{GSH} / \mathrm{GSSG}=[\mathrm{GSH}-(2 \mathrm{GSSG})] / \mathrm{GSSG}$.

\section{Results}

\subsection{The Low Level of MnSOD, Rather than the Deficiency in $\mathrm{Mn}^{2+}$, Causes Oxidative Stress in $\mathrm{Mn}^{2+}$-Deficient Cultures}

To understand whether the oxidative stress in $\mathrm{Mn}^{2+}$-deficient cultures, needed for LIP expression [7] [8], is caused by the deficiency of $\mathrm{Mn}^{2+}$ or by low MnSOD activity or a combination of them, MnSOD silenced mutants (MSC), prepared as previously reported in [18], were used. The MSC mutants were grown in the presence of $\mathrm{Mn}^{2+}$ and molecular oxygen. Significantly higher level of oxidative stress was confirmed in MnSOD silenced mutant (MSC1) in comparison to MnSOD non-silenced mutant (Bar5), by measuring lower GSH/GSSG ratio and higher level of oxidized lipids, in spite of the presence of $\mathrm{Mn}^{2+}$ in the medium. The total GSH levels measured in BAR5 and MSC1 were $142 \mathrm{nM}$ and $270 \mathrm{nM}$, respectively. The GSH/GSSG ratios measured in BAR5 and MSC1 were 1.85 and 1.6, respectively (Figure 1(a), Figure 1(c)). Lipid oxidation was determined by measuring MDA levels in MSC mutants. MDA level in MSC1 was 2.5 times higher than in control BAR5 (Figure 1(d)). Catalase activity was analyzed in protein extracts prepared from the MSC mutants' biomasses. While in BAR5 catalase activity was high (3574 U/mg protein), in MSC1 catalase activity was $1930 \mathrm{U} / \mathrm{mg}$ protein (Figure 1(b)).

\section{2. $\mathrm{H}_{2} \mathrm{O}_{2}$ Is Involved in Triggering of lip- $\mathrm{H} 2$ Expression}

As was reported before, $P$. chrysosporium produce LIP under oxidative stress [6] [7]. Scavenging more molecules of $\mathrm{H}_{2} \mathrm{O}_{2}$ by increasing concentrations of pyruvate in $\mathrm{Mn}^{2+}$-deficient cultures of $P$. chrysosporium resulted in lower lip-H2 expression (Figure 2), indicating the essential dependence role of those molecules in the production of the enzyme by the fungus. At $2 \mathrm{mM}$ pyruvate approximately $70 \%$ of $\mathrm{H}_{2} \mathrm{O}_{2}$ were scavenged (data not shown). 


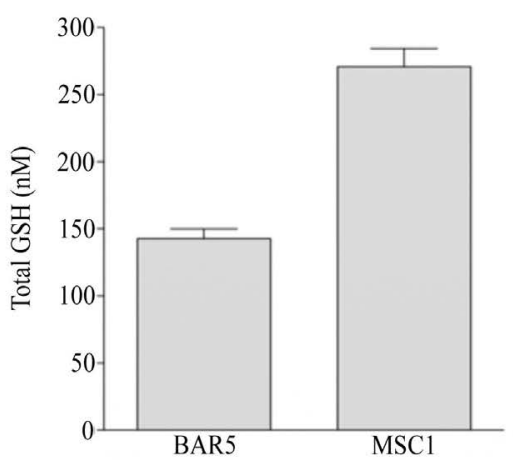

(a)

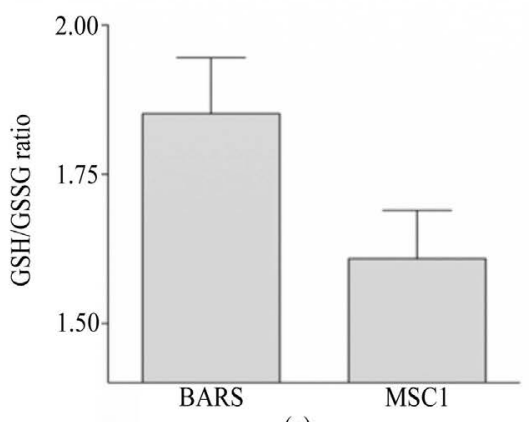

(c)

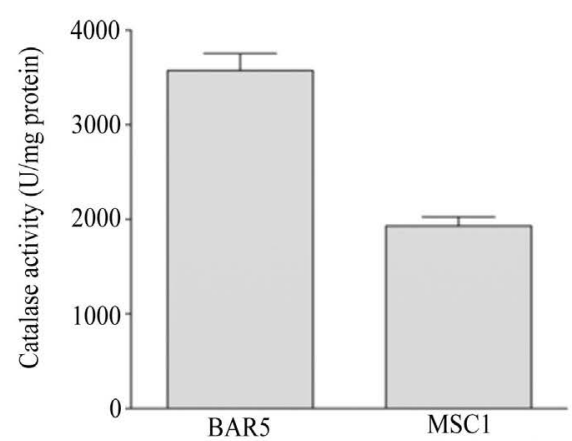

(b)

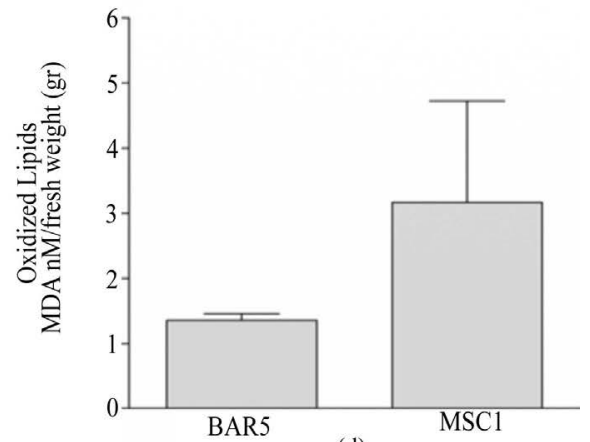

(d)

Figure 1. Measurement of the oxidative stress level in MSC mutants. Total GSH concentrations (a) and GSH/GSSG ratio (c) in extracts, prepared from the MSC mutants' biomasses, were determined by using the Glutathione assay kit (Sigma). The reaction was based on monitoring the production of 5-thio-2-nitrobenzoic acid $\left(\mathrm{TNB}^{2-}\right)$ at $412 \mathrm{~nm}$. The values are means \pm standard deviations of 3 replicates. Lipid damage in MSC mutants (d) was determined in cell extracts of the mutants, grown in oxygenated cultures, by measuring MDA with the LPO-586 kit (OxisResearch). The means \pm SD (error bars) of six replicates are shown. Catalase activity (b) was determined in MSC mutants protein extracts by following the degradation of $\mathrm{H}_{2} \mathrm{O}_{2}$ at $240 \mathrm{~nm}$. The means $\pm \mathrm{SD}$ (error bars) of six replicates are shown.

$\mathrm{Mn}^{2+}$ deficient cultures

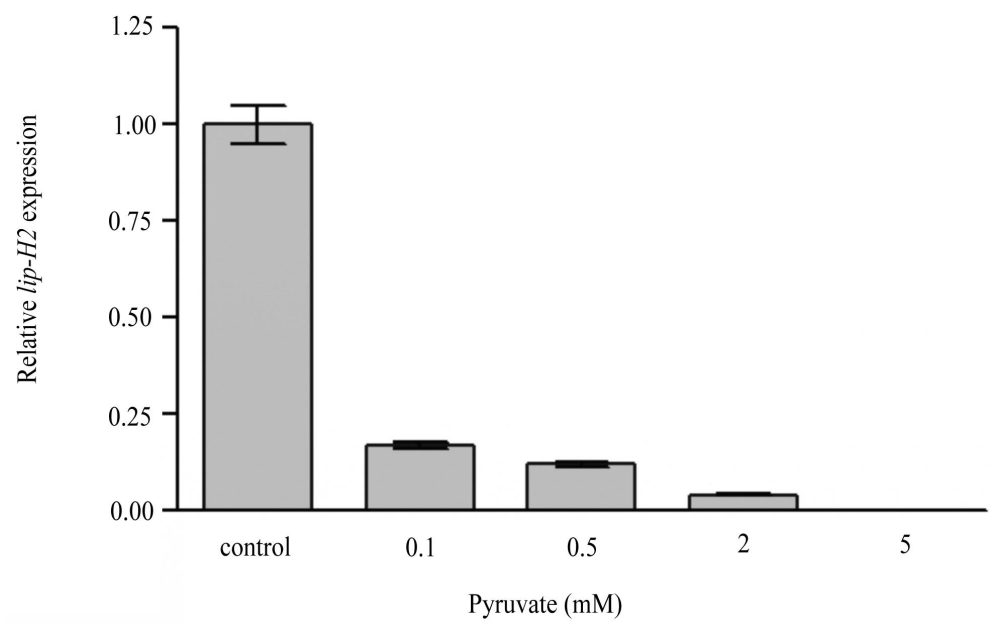

Figure 2. Influence of $\mathrm{H}_{2} \mathrm{O}_{2}$ scavenging on lip- $\mathrm{H} 2$ transcription in $\mathrm{Mn}^{2+}$ deficiency cultures of $P$. chrysosporium. Hydrogen peroxide was scavenged by increased concentrations ( $0-5 \mathrm{mM})$ of pyruvate. The expression of lip- $H 2$ transcripts relative to 18S rRNA gene transcript was measured by real-time PCR. The means \pm SD (error bars) of six replicates are shown. 


\section{3. $\mathrm{H}_{2} \mathrm{O}_{2}$ Production in $\mathrm{Mn}^{2+-}$-Deficient Cultures}

Since one of the consequences of $\mathrm{Mn}^{2+}$ deficiency in $P$. chrysosporium cultures are low levels of MnSOD activity [7] [8], $\mathrm{H}_{2} \mathrm{O}_{2}$ needed for LIP expression should be produced by alternative generators besides MnSOD.

In $\mathrm{Mn}^{2+}$-deficient cultures most of $\mathrm{H}_{2} \mathrm{O}_{2}$ molecules involved in lip- $\mathrm{H} 2$ expression are probably produced by glyoxal oxidase, which was detected as the predominant peroxide producer enzyme expressed in those cultures (Figure 3). CDH level was higher than MnSOD. All the enzymes were similarly expressed in aerated cultures (Figure 3).

\subsection{Role of PKC Activity in Mn'2+-Deficient Cultures}

High production of ROS in $\mathrm{Mn}^{2+}$-deficient cultures may affect lip- $\mathrm{H} 2$ expression by affecting signal transduction pathways enzymes, such as PKC. As presented in Figure 4, PKC activity was significantly lower in $\mathrm{Mn}^{2+}-$ deficient cultures, indicating that for LIP production under high levels of ROS, PKC activity should be low. This was confirmed by inhibition of PKC by different inhibitors in $\mathrm{Mn}^{2+}$-deficient cultures, which provoked the enhancement of lip- $\mathrm{H} 2$ expression (Figure 5).

The PKC inactivation by staurosporin A, calphostin C or $\mathrm{H7}$ caused a significant elevation in lip- $\mathrm{H} 2$ expression, up to 4-fold increase in $\mathrm{Mn}^{2+}$-deficient cultures. The effect of PKC inhibition on lip-H2 expression in aerated cultures was minimal (Figure 5).

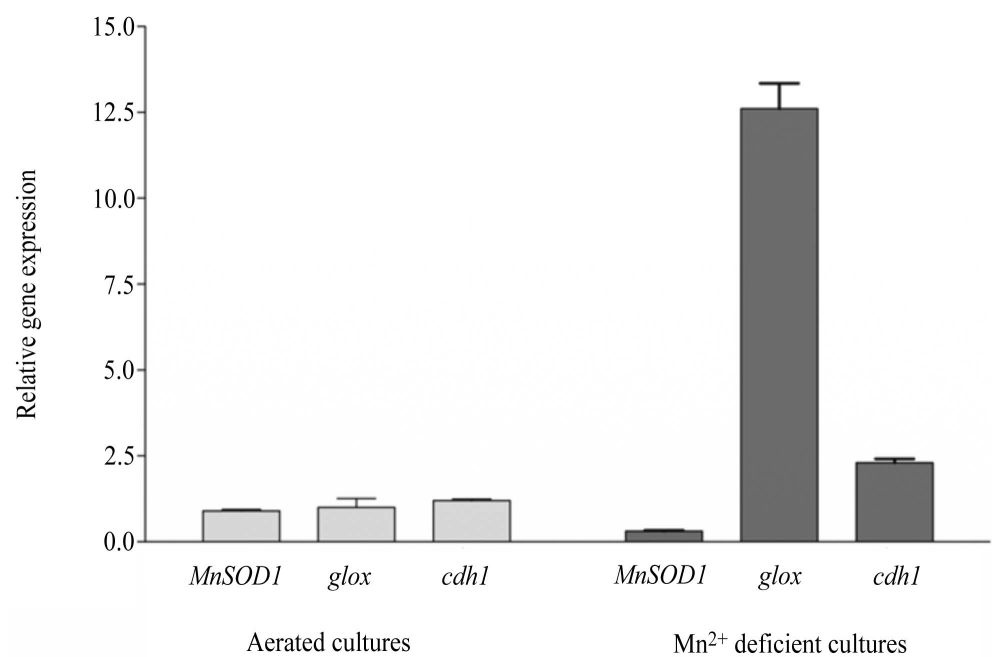

Figure 3. Expression of $\mathrm{H}_{2} \mathrm{O}_{2}$ producer enzymes in $\mathrm{Mn}^{2+}$-deficient high LIPproducing cultures in comparison to aerated low LIP-producing cultures. The expression of MnSOD1, glox and $c d h 1$ transcripts relative to 18S rRNA gene transcript was measured by real-time PCR. The means \pm SD (error bars) of six replicates are shown.

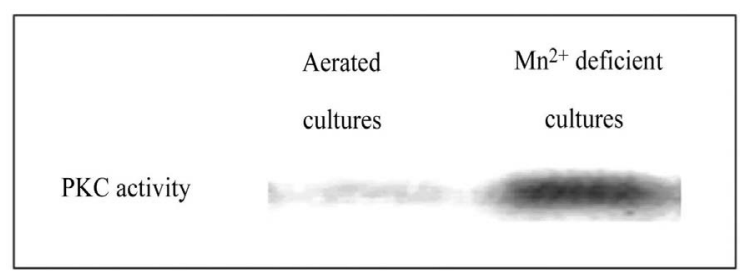

Figure 4. PKC activity in aerated and $\mathrm{Mn}^{2+}$-deficient cultures. Protein samples $(5 \mu \mathrm{g})$ were assayed for PKC activity using the PepTag non-radioactive kit (Promega). The reaction was performed for $30 \mathrm{~min}$ at $30^{\circ} \mathrm{C}$. The nonphosphorylated peptide substrate was then separated from the phosphorylated substrate by electrophoresis on $0.8 \%$ agarose gel. PKC activity was estimated by the fluorescence intensity of the band corresponding to the non-phosphorylated substrate. Intense band represent low activity of PKC. 


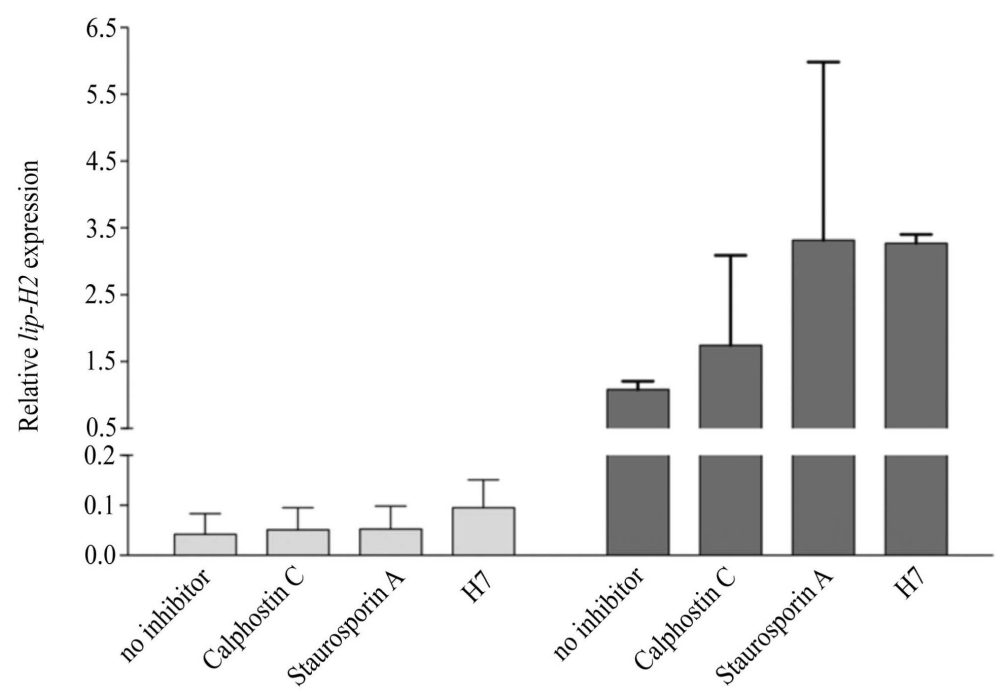

Aerated cultures

$\mathrm{Mn}^{2+}$ deficient cultures

Figure 5. Influence of PKC inhibition on lip- $H 2$ transcription in cell extracts of $\mathrm{Mn}^{2+}$-deficient cultures. The PKC inhibitors (40 ng/ml staurosporin A, 122 $\mathrm{ng} / \mathrm{ml}$ calphostin C or $5 \mu \mathrm{g} / \mathrm{ml} \mathrm{H7}$ ) were added to 120 -h-old aerated and $\mathrm{Mn}^{2+}$ deficient high LIP-producing cultures for $2 \mathrm{~h}$. The expression of lip- $\mathrm{H} 2$ transcripts relative to $18 \mathrm{~S}$ rRNA gene transcript was measured by real-time PCR. The means \pm SD (error bars) of six replicates are shown.

\section{Discussion}

It was previously shown that scavenging of $\mathrm{O}_{2}^{--}$and $\mathrm{H}_{2} \mathrm{O}_{2}$ by tiron and pyruvate, respectively, caused a significant reduction in lip- $\mathrm{H} 2$ expression in oxygenated cultures, indicating that the presence of $\mathrm{O}_{2}^{--}$and $\mathrm{H}_{2} \mathrm{O}_{2}$ is essential for the induction of lip- $\mathrm{H} 2$ expression, probably as precursors for the formation of the $\mathrm{OH}^{*}$ needed to trigger lip- $\mathrm{H} 2$ expression [6] [7] [16]. In this work we show that the presence of $\mathrm{H}_{2} \mathrm{O}_{2}$ is essential for lip- $\mathrm{H} 2$ expression, in $\mathrm{Mn}^{2+}$-deficient cultures, in a dose dependent manner. Despite the low level of MnSOD, the major enzyme responsible for $\mathrm{H}_{2} \mathrm{O}_{2}$ production, in $\mathrm{Mn}^{2+}$-deficient cultures in comparison to oxygenated and aerated cultures [6] [7], there is a sufficient amount of $\mathrm{H}_{2} \mathrm{O}_{2}$, needed for lip- $\mathrm{H} 2$ expression. The $\mathrm{H}_{2} \mathrm{O}_{2}$ present in $\mathrm{Mn}^{2+}$ deficient cultures might be produced by different mechanisms generating less quantity of $\mathrm{H}_{2} \mathrm{O}_{2}$ than MnSOD mechanism. $P$. chrysosporium produce a variety of oxidases that are capable of generating $\mathrm{H}_{2} \mathrm{O}_{2}$, among them are glyoxal oxidase, glucose oxidase, veratryl alcohol oxidase and methanol oxidase [2] [17]. These enzymes may compensate the absence of MnSOD activity in $\mathrm{Mn}^{2+}$-deficient cultures. In this work we show a significantly higher glox expression in high-LIP-producing cultures than low-LIP-producing cultures, indicating a compensation of the deficiency in MnSOD.

Recently, an inverse correlation between ROS/LIP and PKC activities was reported [16]. Significantly low levels of PKC were obtained in oxygenated cultures (containing high ROS levels) relative to aerated cultures (containing low ROS levels). Inhibition or activation of PKC activity in oxygenated cultures was accompanied by a further increase or decrease in lip- $H 2$ expression, respectively. Inhibition of PKC activity in oxygenated cultures also caused an increase in MnSOD1 expression [16]. Here we show that the same correlation between $\mathrm{ROS} / \mathrm{LIP}$ and PKC activities exist in $\mathrm{Mn}^{2+}$-deficient cultures. Significantly lower PKC activity was measured in $\mathrm{Mn}^{2+}$-deficient cultures vs. aerated cultures. Inactivation of PKC activity by staurosporin A, calphostin C or H7 caused significant elevation in lip- $\mathrm{H} 2$, once again indicating that enhancement of LIP production by high levels of ROS is not directly connected with the signal transduction pathway enzyme, PKC, as the direct relationship usually reported for PKC and ROS for other biologic systems [22] [23].

To further understanding the role of $\mathrm{Mn}^{2+}$ deficiency in oxidative stress produced in $\mathrm{Mn}^{2+}$-deficient cultures, MSC mutants previously prepared by using RNAi [18] were used. The MSC mutants have slower growth rate, different MnSOD activity level influenced the fungus growth. Addition of tiron or $\mathrm{H}_{2} \mathrm{O}_{2}$ to the medium im- 
proved its growth rate. The MSC mutants exhibit an unusual hyphal growth (data not shown). MSC1 hyphae are bulbous and thicker at the tip of the hyphae, MSC10 exhibit a highly branched phenotype. Low temperature abolishe this unusual phenotype (data not shown). The involvement of MnSOD in hyphal elongation and branching is known [24].

Mutants having high and low activity of MnSOD [18] were grown in oxygenated conditions in the presence of $\mathrm{Mn}^{2+}$. In those conditions mutants having low activity of MnSOD were subjected to a more severe oxidative stress in comparison to mutants having high activity of MnSOD, which was shown by higher total GSH level, lower GSH/GSSG ratio, higher lipid oxidation and lower level of catalase activity.

Results obtained with BAR5 (non MnSOD silenced mutants) are comparable to that reported in the past for the highest LIP-producing oxygenated cultures [6]. The MnSOD silenced mutants (MSC) might simulate the fungus in cultures grown under $\mathrm{Mn}^{2+}$ deficiency, both having low levels of MnSOD activity. MnSOD silenced mutant which was grown under oxygenated cultures conditions used for wild type, was subjected to severe oxidative stress, despite of the presence of $\mathrm{Mn}^{2+}$. The oxidative stress was even more severe in comparison to wild type oxygenated and $\mathrm{Mn}^{2+}$-deficient cultures [6] [7] due to the direct oxygen flushing to the cultures not containing MnSOD. In aerated cultures, in which MnSOD activity was higher than in $\mathrm{Mn}^{2+}$-deficient cultures due to the presence of $\mathrm{Mn}^{2+}$, a moderate oxidative stress was detected and less LIP was produced [7]. Thus, the lowering of MnSOD by excluding $\mathrm{Mn}^{2+}$, produce an effect similar to that obtained in oxygenated cultures.

\section{Conclusions}

In conclusion, the exclusion of $\mathrm{Mn}^{2+}$ from the culture medium of the fungus probably causes the lowering of MnSOD activity, which in consequence provokes oxidative stress similar to the obtained by oxygen flushing to the medium, needed for the induction of lip- $H 2$ expression and LIP enzyme production.

The oxidative stress mechanism in $\mathrm{Mn}^{2+}$-deficient cultures influences LIP gene in the same way as was showed for oxygenated cultures. Probably, the high levels of ROS, preferentially $\mathrm{OH}^{*}$, generated by reduction of $\mathrm{H}_{2} \mathrm{O}_{2}$, mainly catalyzed by GLOX, inhibit PKC activity as a regulatory strategy in the cells, inducing LIP.

All the results obtained in this work can contribute to the understanding of LIP regulation in P. chysosporium.

\section{References}

[1] de Jong, E. (1993) Physiological Roles and Metabolism of Fungal Aryl Alcohols. Wageningen Agricultural University, Wageningen.

[2] Gold, M.H. and Alic, M. (1993) Molecular Biology of the Lignin-Degrading Basidiomycete Phanerochaete chrysosporium. Microbiological Reviews, 57, 605-622.

[3] Cullen, D. (2002) Molecular Genetics of Lignin-Degrading Fungi and Their Applications in Organopollutant Degradation. In: The Mycota: A Comprehensive Treatise on Fungi as Experimental Systems for Basic and Applied Research, Vol. 11, Agricultural Applications, Kempken, Springer, Berlin, 71-90.

[4] Kirk, T.K. and Farrell, R.L. (1987) Enzymatic “Combustion”: The Microbial Degradation of Lignin. Annual Review of Microbiology, 41, 465-505. http://dx.doi.org/10.1146/annurev.mi.41.100187.002341

[5] Tien, M. and Kirk, T.K. (1984) Lignin-Degrading Enzyme from Phanerochaete chrysosporium: Purification, Characterization, and Catalytic Properties of a Unique $\mathrm{H}_{2} \mathrm{O}_{2}$-Requiring Oxygenase. Proceedings of the National Academy of Sciences of the United States of America, 81, 2280-2284. http://dx.doi.org/10.1073/pnas.81.8.2280

[6] Belinky, P.A., et al. (2003) Reactive Oxygen Species and Induction of Lignin Peroxidase in Phanerochaete chrysosporium. Applied and Environmental Microbiology, 69, 6500-6506. http://dx.doi.org/10.1128/AEM.69.11.6500-6506.2003

[7] Belinky, P.A., Flikshtein, N. and Dosoretz, C.G. (2006) Induction of Lignin Peroxidase via Reactive Oxygen Species in Manganese-Deficient Cultures of Phanerochaete chrysosporium. Enzyme and Microbial Technology, 39, $222-228$. http://dx.doi.org/10.1016/j.enzmictec.2005.10.023

[8] Rothschild, N., et al. (1999) Manganese Deficiency Can Replace High Oxygen Levels Needed for Lignin Peroxidase Formation by Phanerochaete chrysosporium. Applied and Environmental Microbiology, 65, 483-488.

[9] Boominatan, K. and Reddy, C.A. (1992) cAMP-Mediated Differential Regulation of Lignin Peroxidase and ManganeseDependent Peroxidase Production in the White-Rot Basidiomycete Phanerochaete chrysosporium. Proceedings of the National Academy of Sciences of the United States of America, 89, 5586-5590. http://dx.doi.org/10.1073/pnas.89.12.5586

[10] Bar-Lev, S.S. and Kirk, T.K. (1981) Effects of Molecular Oxygen on Lignin Degradation by Phanerochaete chrysosporium. Biochemical and Biophysical Research Communications, 99, 373-378. 
http://dx.doi.org/10.1016/0006-291X(81)91755-1

[11] Dosoretz, C.G., Chen, A.H.C. and Grethlein, H.E. (1990) Effect of Oxygenation Conditions on Submerged Cultures of Phanerochaete chrysosporium. Applied Microbiology and Biotechnology, 34, 131-137. http://dx.doi.org/10.1007/BF00170937

[12] Dosoretz, C.G., Rothschild, N. and Hadar, Y. (1993) Overproduction of Lignin Peroxidase by Phanerochaete chrysosporium (BKM-F-1767) under Nonlimiting Nutrient Conditions. Applied and Environmental Microbiology, 59, 19191926.

[13] Dosoretz, C.G. and Grethlein, H.E. (1991) Physiological Aspects of the Regulation of Extracellular Enzymes of Phanerochaete chrysosporium. Applied Biochemistry and Biotechnology, 28-29, 253-265. http://dx.doi.org/10.1007/BF02922605

[14] Forney, L.J., Reddy, C.A., Tien, M. and Aust, S.D. (1982) The Involvement of Hydroxyl Radical Derived from Hydrogen Peroxide in Lignin Degradation by the White Rot Fungus Phanerochaete chrysosporium. Journal of Biological Chemistry, 257, 11455-11462.

[15] Tien, M. and Tu, C.P. (1987) Cloning and Sequencing of a cDNA for a Ligninase from Phanerochaete chrysosporium. Nature, 326, 520-523. http://dx.doi.org/10.1038/326520a0

[16] Matityahu, A., Hadar, Y. and Belinky, P.A. (2010) Involvement of Protein Kinase C in Lignin Peroxidase Expression in Oxygenated Cultures of the White Rot Fungus Phanerochaete chrysosporium. Enzyme and Microbial Technology, 47, 59-63. http://dx.doi.org/10.1016/j.enzmictec.2010.05.002

[17] Cullen, D. and Kersten, P.J. (2004) Enzymology and Molecular Biology of Lignin Degradation. In: The Mycota III: Biochemistry and Molecular Biology, Springer-Verlag, Berlin, 249-273.

[18] Matityahu, A., Hadar, Y., Dosoretz, C.G. and Belinky, P.A. (2008) Gene Silencing by RNA Interference in the White Rot Fungus Phanerochaete chrysosporium. Applied and Environmental Microbiology, 74, 5359-5365. http://dx.doi.org/10.1128/AEM.02433-07

[19] Tien, M. and Kirk, T.K. (1988) Lignin Peroxidase of Phanerochaete chrysosporium. Methods in Enzymology, 161, 238-249. http://dx.doi.org/10.1016/0076-6879(88)61025-1

[20] Rothschild, N., Hadar, Y. and Dosoretz, C.G. (1995) Ligninolytic System Formation by Phanerochaete chrysosporium in Air. Applied and Environmental Microbiology, 61, 1833-1838.

[21] Lee, J.S., Hah, Y.C. and Roe, J.H. (1993) The Induction of Oxidative Enzymes in Streptomyces coelicolor upon Hydrogen Peroxide Treatment. Journal of General Microbiology, 139, 1013-1018. http://dx.doi.org/10.1099/00221287-139-5-1013

[22] Schmitz, H.P. and Heinisch, J.J. (2003) Evolution, Biochemistry and Genetics of Protein Kinase C in Fungi. Current Genetics, 43, 245-254. http://dx.doi.org/10.1007/s00294-003-0403-6

[23] Maher, P. (2001) How Protein Kinase C Activation Protects Nerve Cells from Oxidative Stress-Induced Cell Death. Journal of Neuroscience, 21, 2929-2938.

[24] Georgiou, C.D., Patsoukis, N., Papapostolou, I. and Zervoudakis, G. (2006) Sclerotial Metamorphosis in Filamentous Fungi Is Induced by Oxidative Stress. Integrative and Comparative Biology, 46, 691-712.

http://dx.doi.org/10.1093/icb/icj034 
Scientific Research Publishing (SCIRP) is one of the largest Open Access journal publishers. It is currently publishing more than 200 open access, online, peer-reviewed journals covering a wide range of academic disciplines. SCIRP serves the worldwide academic communities and contributes to the progress and application of science with its publication.

Other selected journals from SCIRP are listed as below. Submit your manuscript to us via either submit@scirp.org or Online Submission Portal.
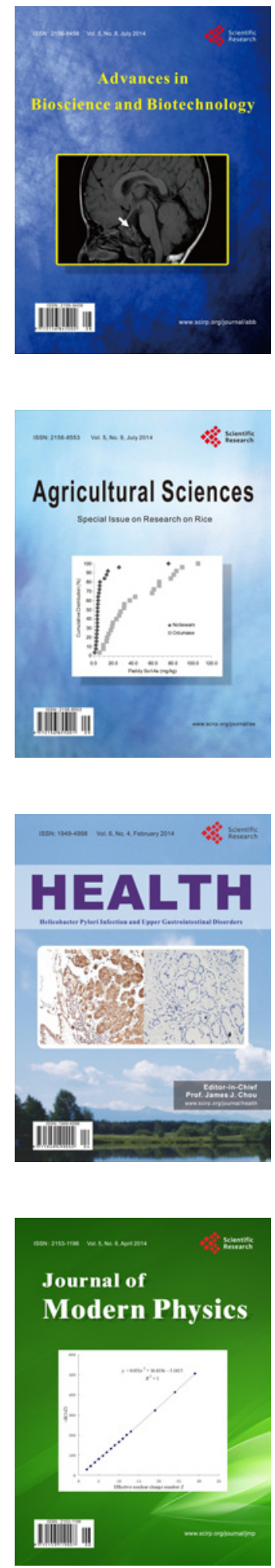
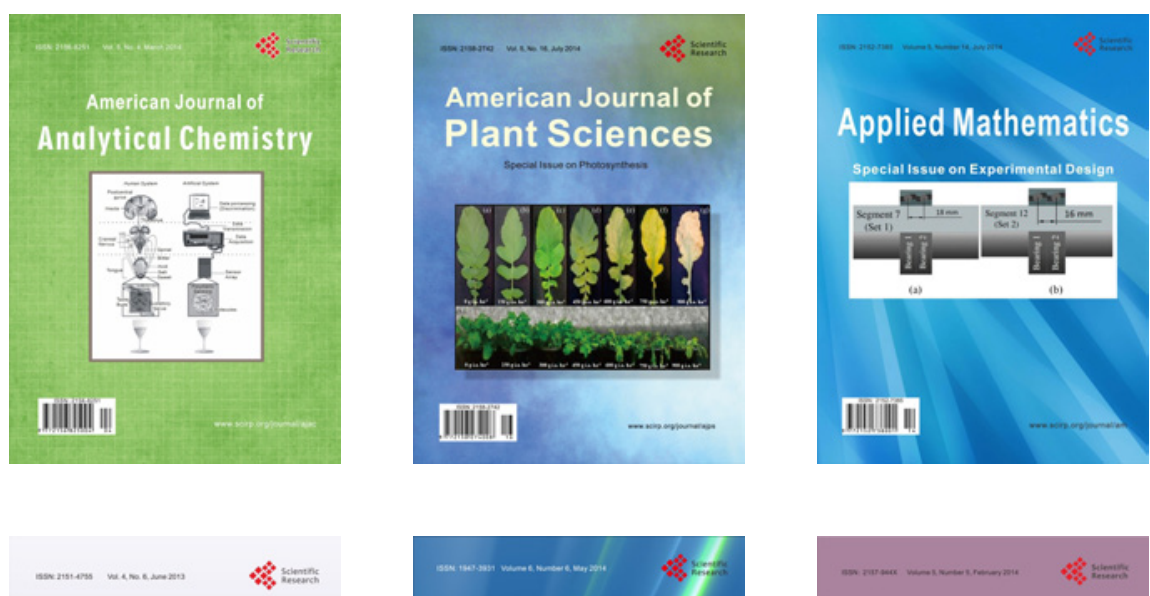

Creative Education
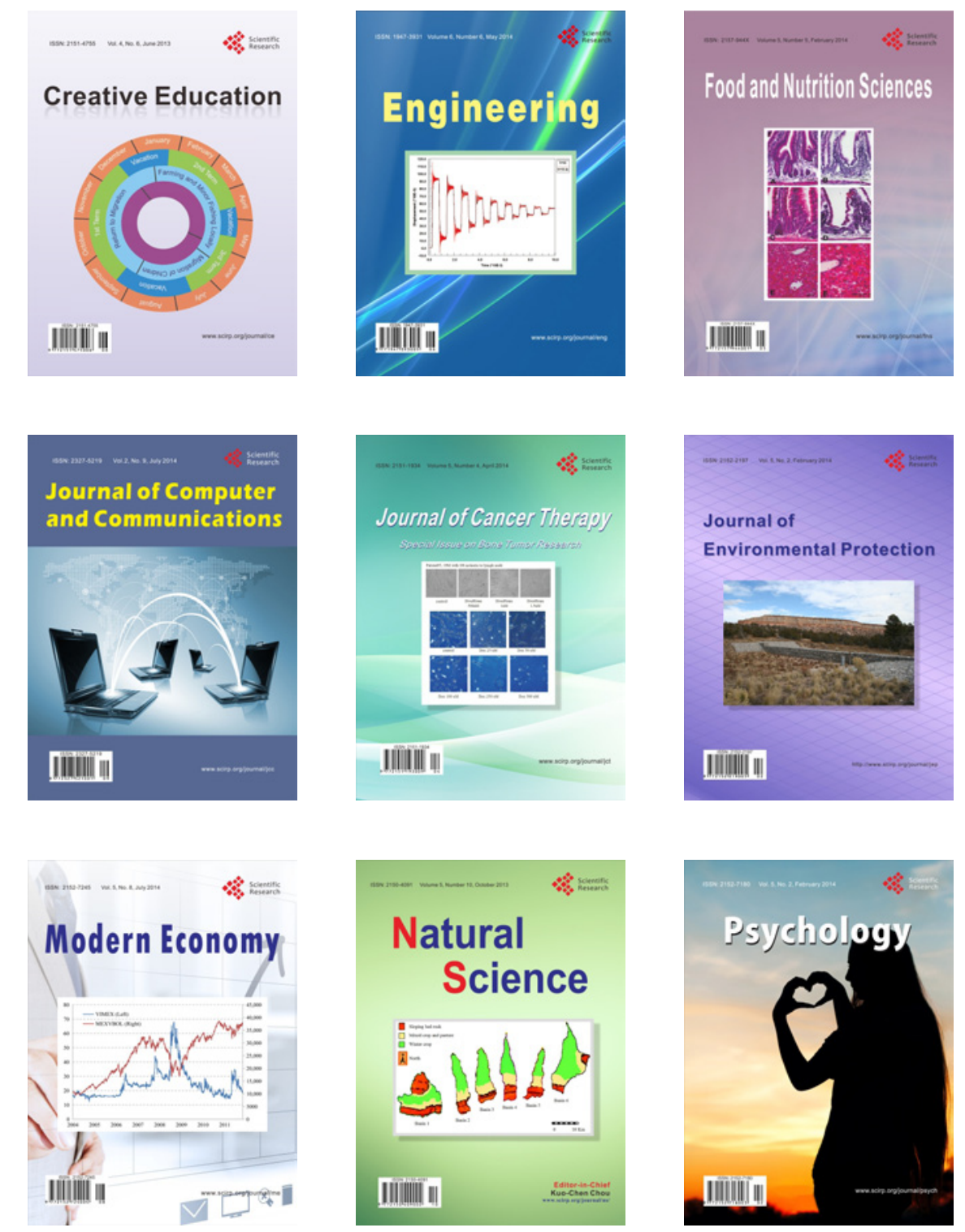